DF/IST-1.2007

\title{
Spontaneous Symmetry Breaking in the Bulk as a Localization Mechanism of Fields on the Brane
}

\author{
Orfeu Bertolami* and Carla Carvalhd $\dagger$ \\ Departamento de Física, Instituto Superior Técnico \\ Avenida Rovisco Pais 1, 1049-001 Lisboa, Portugal
}

\begin{abstract}
We consider real and complex scalar fields non-minimally coupled to gravity in the bulk spacetime and study their impact on the brane upon acquiring a non-vanishing vacuum expectation value. When examining the case of the complex scalar field, a minimally coupled $U(1)$ gauge field is also considered so that spontaneous symmetry breaking can take place. Particular attention is paid to the terms arising from the junction conditions, which act towards reinforcing the mechanism of spontaneous symmetry breaking. We find that the embedding of a braneworld universe in a bulk spacetime endowed with matter fields can induce this mechanism at very high energies, which implies the localization on the brane of the bulk fields.
\end{abstract}

\footnotetext{
*Also at Centro de Física dos Plasmas, Instituto Superior Técnico; Email address: orfeu@cosmos.ist.utl.pt ${ }^{\dagger}$ Also at Centro de Física dos Plasmas, Instituto Superior Técnico; Email address: ccarvalho@ist.edu
} 


\section{INTRODUCTION}

The breaking of geometrical symmetries or internal symmetries, such as those associated with gauge invariance, is a crucial ingredient in physics. This breaking plays a central role both in condensed matter physics and in quantum field theory. In particular, the phenomenological consistency of the electroweak unification relies fundamentally on the breaking of a gauge symmetry. It is well known that some important features of the symmetry breaking mechanism are altered once the coupling to gravity is considered. For the case of scalar fields this occurs via a non-minimal coupling with the scalar curvature. Such coupling may lead to important physical consequences, as it affects the positivity of the Einstein tensor and the stability of theories coupled to gravity [1]. It is worth stressing that the positivity of the Einstein tensor is a condition of the well-known singularity theorems for gravity. A non-minimal coupling plays also a crucial role in some alternative gravity models, commonly referred to as induced gravity [2], as it is through this coupling that the mechanism of symmetry breaking can generate a gravitational coupling and an induced cosmological constant.

In this work we shall consider such non-minimally coupled scalar field models in the context of braneworlds. The two bulk fields, namely gravity and the scalar field, must be allowed to interact in the five-dimensional spacetime and not solely on the boundary. The simplest such interaction couples the scalar curvature to the scalar field. Notice that in string theory, for instance, the couplings to the dilaton at the affective action level involve instead an overall exponential function in the s-parametrization and derivative terms [3].

Bulk scalar fields have been previously discussed in various studies of non-vanishing vacuum bulk field configurations, in both one [4, 5] and two-brane setups [6, 7]. Here we study real and complex scalar fields in the bulk spacetime and examine their implication for the mechanism of spontaneous symmetry breaking (SSB) on the brane upon acquiring a non-vanishing bulk vacuum expectation value (vev). When studying the case of the complex scalar field, we also consider a minimally coupled $U(1)$ gauge field so that spontaneous breaking of the gauge symmetry can take place. New terms arising from the junction conditions will contribute to the equations induced on the brane and entail consequences in the induced SSB mechanism. Since the masses induced by the brane mediated SSB mechanism are of order the four-dimensional Planck mass, the range of the induced interactions become short about the brane, suggesting the localization of the bulk fields on the brane. 
This paper consists also of a preliminary study of the possibility of using spontaneous symmetry breaking as a means for localizing matter on the brane. In the Goldstone mechanism, a mass is attained in the direction along which the system undergoes spontaneous symmetry breaking. The consequent gain in inertia would be expected to prevent the motion of matter along that direction and thus to constrain matter to stay on the orthogonal directions, which would define the brane comprising the observable universe. We aim to further pursue this idea in a follow-up publication.

Here we establish how to derive brane quantities from bulk quantities by adopting Fermi normal coordinates with respect to the directions parallel to the brane and continuing into the bulk using the Gauss normal prescription. After deriving the equations of motion in the bulk, we project them parallel and orthogonal to the surface of the brane. The brane is assumed to be a distribution of $Z_{2}$-symmetric stress-energy about a shell of thickness $2 \delta$ in the limit $\delta \rightarrow 0$. Derivatives of quantities discontinuous across the brane will generate singular distributions on the brane which relate to the localization of the stress-energy. This relation is encompassed by the matching conditions across the brane obtained by the integration of the corresponding equation of motion in the direction normal to the brane. The matching conditions provide the boundary conditions on the brane for the bulk fields, thus constraining the parallel projected equations to produce the induced equations on the brane. Then we discuss the SSB mechanism in the context of a braneworld universe embedded in a five-dimensional anti-de Sitter $\left(A d S_{5}\right)$ bulk spacetime. Spontaneous symmetry breaking is treated by assuming that the bulk scalar fields acquire a non-vanishing expectation value, which induces on the brane the breaking of the existing gauge symmetry when the scalar field is minimally coupled to a gauge field.

Notice that the formalism employed here has been developed earlier [8] and has moreover been used to study a vector field coupled non-minimally to gravity in order to examine the implications on the brane of the spontaneous breaking of Lorentz symmetry in the bulk [9]. In what follows, $d$ stands for the total number of space dimensions.

This paper is organized as follows. In Section \I we work out the case of a bulk scalar field coupled non-minimally to gravity. In Section III we endow the scalar field with a $U(1)$ charge, introduce an additional gauge field and implement the SSB mechanism. This requirement is of particular relevance since, once the gauge fields acquire a mass, they become short range fields and must thus become confined to the brane. For each case we derive the effective equations of motion for the bulk fields as induced on the brane. 


\section{SCALAR FIELD}

In this section we consider a bulk real scalar field $\phi$, which contributes to the purely gravitational Lagrangian density for $A d S_{5}$ spacetime with canonical kinetic and potential terms and with a $\phi$-graviton interaction term as follows

$$
\mathcal{L}=\frac{1}{\kappa_{(5)}^{2}} R-2 \Lambda+\xi \phi^{2} R-\frac{1}{2} g^{\mu \nu}\left(\nabla_{\mu} \phi\right)\left(\nabla_{\nu} \phi\right)-V\left(\phi^{2}\right) .
$$

Here, $\kappa_{(5)}^{2}=8 \pi G_{N(5)}=1 / M_{P l}^{3}$ is the five-dimensional gravitational coupling constant and $\xi$ is a dimensionless coupling constant which measures the non-minimal interaction. In the cosmological constant term $\Lambda=\Lambda_{(5)}+\Lambda_{(4)}$ we have included both the bulk vacuum value $\Lambda_{(5)}$ and that of the brane $\Lambda_{(4)}$, described by a brane tension $\sigma$ localized at the position of the brane, $\Lambda_{(4)}=\sigma \delta(N)$.

A distinct feature of our model in comparison with more standard braneworld approaches is the non-minimal coupling of the scalar field to gravity via the Ricci scalar. As mentioned in the introduction, this type of coupling term appears in alternative gravity models and offers an interesting possibility of relating Newton's constant, the cosmological constant and the mechanism of SSB (see e.g. [1, 10] and references therein). Given the relevance of these issues, examining their relationship in the context of the braneworlds is quite logical since in these models the Newton's constant, the five-dimensional cosmological constant and the brane tension are entangled. In this framework, the mechanism of SSB allows to relate the mentioned quantities with the process of generation of mass.

\section{A. The induced dynamics}

First we derive the equations of motion for both the scalar field and the graviton as measured by an observer localized on and confined to the brane. This follows closely the procedure developed in Ref. [9] for a vector field and in particular uses the results presented in the appendix therein.

By varying the action with respect to the metric, we obtain the Einstein equation in the bulk

$$
\left(\frac{1}{\kappa_{(5)}^{2}}+\xi \phi^{2}\right) G_{\mu \nu}+\Lambda g_{\mu \nu}=\frac{1}{2} T_{\mu \nu}^{(\phi)}+\xi \Sigma_{\mu \nu}^{(\phi)}
$$

where

$$
T_{\mu \nu}^{(\phi)}=\left(\nabla_{\mu} \phi\right)\left(\nabla_{\nu} \phi\right)+g_{\mu \nu}\left[-\frac{1}{2} g^{\alpha \beta}\left(\nabla_{\alpha} \phi\right)\left(\nabla_{\beta} \phi\right)-V\left(\phi^{2}\right)\right]
$$


is the stress-energy tensor associated with $\phi$ and

$$
\Sigma_{\mu \nu}^{(\phi)}=\nabla_{\mu} \nabla_{\nu} \phi^{2}-g_{\mu \nu} g^{\alpha \beta} \nabla_{\alpha} \nabla_{\beta} \phi^{2}
$$

is the contribution from the interaction term. We note the $\phi$-dependence of the fivedimensional gravitational coupling constant akin to that of the Brans-Dicke formulation. For the equation of motion for the $\phi$ field, obtained by varying the action with respect to $\phi$, we find that

$$
g^{\mu \nu} \nabla_{\mu} \nabla_{\nu} \phi-\frac{\partial V}{\partial \phi}+2 \xi \phi R=0
$$

We now proceed to project the equations parallel and orthogonal to the surface of the brane, finding for the stress-energy tensor $\mathbf{T}$ that

$$
\begin{aligned}
T_{A B}^{(\phi)} & =\left(\nabla_{A} \phi\right)\left(\nabla_{B} \phi\right)+g_{A B}\left[-\frac{1}{2}\left[\left(\nabla_{C} \phi\right)^{2}+\left(\nabla_{N} \phi\right)^{2}\right]-V\left(\phi^{2}\right)\right], \\
T_{A N}^{(\phi)} & =\left(\nabla_{A} \phi\right)\left(\nabla_{N} \phi\right), \\
T_{N N}^{(\phi)} & =\left(\nabla_{N} \phi\right)\left(\nabla_{N} \phi\right)+g_{N N}\left[-\frac{1}{2}\left[\left(\nabla_{C} \phi\right)^{2}+\left(\nabla_{N} \phi\right)^{2}\right]-V\left(\phi^{2}\right)\right],
\end{aligned}
$$

and similarly for the source tensor $\boldsymbol{\Sigma}$ that

$$
\begin{aligned}
\Sigma_{A B}^{(\phi)} & =\left(\nabla_{A} \nabla_{B}+K_{A B} \nabla_{N}\right) \phi^{2}-g_{A B}\left(\nabla_{C}^{2}+\nabla_{N}^{2}+K \nabla_{N}\right) \phi^{2}, \\
\Sigma_{A N}^{(\phi)} & =\left(\nabla_{A} \nabla_{N}-K_{A B} \nabla_{B}\right) \phi^{2}, \\
\Sigma_{N N}^{(\phi)} & =\nabla_{N} \nabla_{N} \phi^{2}-g_{N N}\left(\nabla_{C}^{2}+\nabla_{N}^{2}+K \nabla_{N}\right) \phi^{2} .
\end{aligned}
$$

Equating the $(A B)$ components of the decomposition of the Einstein tensor and of the source terms from the scalar field $\phi$, we find for the Einstein equation parallel projected on to the brane that

$$
\begin{aligned}
& \left(\frac{1}{\kappa_{(5)}^{2}}+\xi \phi^{2}\right)\left[G_{A B}^{(i n d)}+2 K_{A C} K_{C B}-K_{A B} K-K_{A B, N}-\frac{1}{2} g_{A B}\left(-K_{C D} K_{D C}-K^{2}-2 K_{, N}\right)\right] \\
= & \frac{1}{2}\left(\nabla_{A} \phi\right)\left(\nabla_{B} \phi\right)+\frac{1}{2} g_{A B}\left[-\frac{1}{2}\left[\left(\nabla_{C} \phi\right)^{2}+\left(\nabla_{N} \phi\right)^{2}\right]-V\left(\phi^{2}\right)\right]-g_{A B} \Lambda \\
+ & \xi\left[\left(\nabla_{A} \nabla_{B}+K_{A B} \nabla_{N}\right) \phi^{2}-g_{A B}\left(\nabla_{C}^{2}+\nabla_{N}^{2}+K \nabla_{N}\right) \phi^{2}\right] .
\end{aligned}
$$

To obtain the matching condition for the extrinsic curvature across the brane, we integrate the $(A B)$ component of the Einstein equation in the coordinate normal to the brane. From the $Z_{2}$-symmetry it follows that $\phi(-\delta)=\phi(+\delta)$ and consequently that $\nabla_{N} \phi(-\delta)=-\nabla_{N} \phi(+\delta)$. Moreover, continuity of the induced metric across the brane 
$g_{A B}(-\delta)=g_{A B}(+\delta)$ implies that $K_{A B}(-\delta)=-K_{A B}(+\delta)$. We then obtain for the $(A B)$ matching condition

$$
\begin{aligned}
& \int_{-\delta}^{+\delta} d N\left(\frac{1}{\kappa_{(5)}^{2}}+\xi \phi^{2}\right)\left(-K_{A B, N}+g_{A B} K_{, N}\right) \\
= & \int_{-\delta}^{+\delta} d N\left[-g_{A B} \Lambda_{(4)}+\xi\left(\left(K_{A B}-g_{A B} K\right) \nabla_{N} \phi^{2}-g_{A B} \nabla_{N}^{2} \phi^{2}\right)\right],
\end{aligned}
$$

which yields

$$
\left(\frac{1}{\kappa_{(5)}^{2}}+\xi \phi^{2}\right)\left(-K_{A B}+g_{A B} K\right)=g_{A B}\left(-\sigma-\xi \nabla_{N} \phi^{2}\right)
$$

for $\phi^{2}$ even about the position of the brane. These provide boundary conditions for ten of the fifteen degrees of freedom. Five additional boundary conditions are provided by the matching conditions from the $(A N)$ and $(N N)$ components of the projected Einstein equations. From inspection of the $(A N)$ components we note that

$$
\begin{aligned}
G_{A N}=K_{A B ; B}-K_{; A} & =-\nabla_{B}\left(\int d N G_{A B}\right) \\
& =-\nabla_{B}\left(\int d N \frac{T_{A B}^{(\phi)}}{1 / \kappa_{(5)}^{2}+\xi \phi^{2}}\right)=-\nabla_{B} \mathcal{T}_{A B}^{(\phi)}=0,
\end{aligned}
$$

which vanishes due to conservation of the induced stress-energy $\mathcal{T}_{A B}^{(\phi)}$ on the brane, as read off of Eq. (10). This condition constrains four degrees of freedom. The $(N N)$ component of the Einstein equation

$$
\begin{aligned}
& \left(\frac{1}{\kappa_{(5)}^{2}}+\xi \phi^{2}\right)\left[-R^{(i n d)}-K_{C D} K_{C D}+K^{2}\right]+\Lambda_{(5)} \\
= & \frac{1}{2}\left[\frac{1}{2}\left(\nabla_{N} \phi\right)\left(\nabla_{N} \phi\right)-\frac{1}{2}\left(\nabla_{C} \phi\right)\left(\nabla_{C} \phi\right)-V\left(\phi^{2}\right)\right]-\xi\left(\nabla_{C}^{2}+K \nabla_{N}\right) \phi^{2}
\end{aligned}
$$

consists of the remaining constraint. Substituting the $(A B)$ matching condition, Eq. (10), back in the $(A B)$ Einstein equation, Eq. (8) , we find for the Einstein equation induced on the brane

$$
\begin{aligned}
& \left(\frac{1}{\kappa_{(5)}^{2}}+\xi \phi^{2}\right)\left[G_{A B}^{(i n d)}+2 K_{A C} K_{C B}-K_{A B} K-\frac{1}{2} g_{A B}\left(-K_{C D} K_{D C}-K^{2}\right)\right]+g_{A B} \Lambda_{(5)} \\
= & \frac{1}{2}\left(\nabla_{A} \phi\right)\left(\nabla_{B} \phi\right)+\frac{1}{2} g_{A B}\left[-\frac{1}{2}\left(\nabla_{C} \phi\right)^{2}-\frac{1}{2}\left(\nabla_{N} \phi\right)^{2}-V\right]+\xi\left(\nabla_{A} \nabla_{B}-g_{A B} \nabla_{C}^{2}\right) \phi^{2} .
\end{aligned}
$$

Similarly, we expand the equation of motion for the scalar field $\phi$

$$
\left[g^{A B}\left(\nabla_{A} \nabla_{B}+K_{A B} \nabla_{N}\right)+\nabla_{N} \nabla_{N}\right] \phi-\frac{\partial V}{\partial \phi}
$$




$$
+2 \xi \phi\left(R^{(i n d)}-K_{A B} K_{B A}-K^{2}-2 K_{, N}\right)=0 .
$$

To obtain the matching condition for $\phi$ across the brane, we integrate in the $N$ coordinate discarding all derivatives other than along $N$

$$
\int_{-\delta}^{+\delta} d N\left[K \nabla_{N} \phi+\nabla_{N} \nabla_{N} \phi-4 \xi \phi K_{, N}\right]=0 .
$$

If we assume $Z_{2}$-symmetry across the brane, the matching condition for $\phi$ becomes

$$
\nabla_{N} \phi-4 \xi K \phi=0
$$

Substituting Eq. (16) back into Eq. (14), we obtain for the propagation of $\phi$ on the brane that

$$
g^{A B} \nabla_{A} \nabla_{B} \phi-\frac{\partial V}{\partial \phi}+2 \xi \phi\left[R^{(i n d)}-K_{A B} K_{B A}+(1+8 \xi) K^{2}\right]=0 .
$$

Moreover, equating the matching conditions for the extrinsic curvature, Eq. (10), and for the scalar field $\phi$, Eq. (16), we can solve for $K_{A B}$ and $\nabla_{N} \phi$ to find that

$$
\begin{aligned}
K_{A B} & =-\left.g_{A B} \sigma \frac{1 /(d-1)}{1 / \kappa_{(5)}^{2}+\xi \phi^{2}[1+8 \xi d /(d-1)]}\right|_{N=0}, \\
\left.\nabla_{N} \phi\right|_{N=0} & =-\left.4 \xi \phi \sigma \frac{d /(d-1)}{1 / \kappa_{(5)}^{2}+\xi \phi^{2}[1+8 \xi d /(d-1)]}\right|_{N=0} .
\end{aligned}
$$

We substitute Eq. (16) for $\nabla_{N} \phi$ and Eq. (17) for $\nabla_{C}^{2} \phi$ in the $(N N)$ component of the Einstein equation, Eq. (12), to find for $R^{(i n d)}$ that

$$
\begin{gathered}
\left(\frac{1}{\kappa_{(5)}^{2}}+\xi \phi^{2}(1+4 \xi)\right) R^{(i n d)}=\left(\frac{1}{4}+2 \xi\right)\left(\nabla_{C} \phi\right)^{2}+\frac{1}{2} V+2 \xi \phi \frac{\partial V}{\partial \phi} \\
+\Lambda_{(5)}-K_{C D} K_{C D}\left(\frac{1}{\kappa_{(5)}^{2}}+\xi \phi^{2}(1-4 \xi)\right)+K^{2}\left(\frac{1}{\kappa_{(5)}^{2}}+\xi \phi^{2}\left(1-32 \xi^{2}\right)\right) .
\end{gathered}
$$

Substituting now in Eq. (17) we find for the equation of motion for $\phi$ induced on the brane that

$$
\begin{aligned}
& g^{A B} \nabla_{A} \nabla_{B} \phi-\frac{\partial V}{\partial \phi}\left(\frac{1}{\kappa_{(5)}^{2}}+\xi \phi^{2}\right) \frac{1}{1 / \kappa_{(5)}^{2}+\xi \phi^{2}(1+4 \xi)} \\
+ & {\left[\left(\frac{1}{4}+2 \xi\right)\left(\nabla_{C} \phi\right)^{2}+\frac{1}{2} V+\Lambda_{(5)}\right] \frac{2 \xi \phi}{1 / \kappa_{(5)}^{2}+\xi \phi^{2}(1+4 \xi)} } \\
+ & K^{2} \frac{2 \xi \phi}{1 / \kappa_{(5)}^{2}+\xi \phi^{2}(1+4 \xi)}\left[\frac{1}{\kappa_{(5)}^{2}}\left(-\frac{2}{d}+2+8 \xi\right)+\xi \phi^{2}\left(-\frac{2}{d}+2+12 \xi\right)\right]=0 .
\end{aligned}
$$


Similarly, substituting in Eq. (13) we find for the Einstein equation induced on the brane that

$$
\begin{aligned}
G_{A B}^{(i n d)}= & \left(\frac{1}{\kappa_{(5)}^{2}}+\xi \phi^{2}\right)^{-1}\left[\left(\frac{1}{2}+2 \xi\right)\left(\nabla_{A} \phi\right)\left(\nabla_{B} \phi\right)+2 \xi \phi \nabla_{A} \nabla_{B} \phi\right] \\
- & g_{A B}\left[\left(\frac{1}{4}+2 \xi\right)\left(\nabla_{C} \phi\right)^{2}+\frac{1}{2} V+2 \xi \phi \frac{\partial V}{\partial \phi}+\Lambda_{(5)}\right] \frac{1}{1 / \kappa_{(5)}^{2}+\xi \phi^{2}(1+4 \xi)} \\
- & g_{A B} K^{2}\left[\left(\frac{1}{\kappa_{(5)}^{2}}+\xi \phi^{2}\right) \frac{d^{2}-d+4}{2 d^{2}}+(2 \xi \phi)^{2}\left(\frac{-d^{2}+3 d+4}{2 d^{2}}-8 \xi\right)\right] \\
& \times \frac{1}{1 / \kappa_{(5)}^{2}+\xi \phi^{2}(1+4 \xi)} .
\end{aligned}
$$

Using the equations derived above, we realize the case where the scalar field acquires a non-vanishing vev $\langle\phi\rangle$ which minimizes the effective potential. This can induce spontaneous symmetry breaking when the scalar field is coupled to a gauge field, thus endowing the latter with a mass, as will be discussed in Section [II] Here, however, a non-vanishing vev will entail a change in the effective cosmological constant and in the effective mass of the scalar field. Moreover, once the scalar field acquires a vev, no direction on the brane can be selected, which implies that $\nabla_{A}\langle\phi\rangle=0$. Consequently, Lorentz symmetry breaking cannot take place in the presence of a bulk scalar field only (see Ref. [9] for the case of an explicit violation of Lorentz symmetry due to a non-vanishing vev for a vector field).

We can read off of the induced Einstein equation the effective cosmological constant, which would comprise all the terms proportional to the induced metric which do not vanish when all the contributions from the matter fields vanish. However, in the case that the matter fields acquire a non-vanishing vev, the effective cosmological constant will contain the contribution of the matter fields at the corresponding non-vanishing value. It follows that

$$
\begin{gathered}
\Lambda_{e f f}\left(\frac{1}{\kappa_{(5)}^{2}}+\xi\langle\phi\rangle^{2}(1+4 \xi)\right)=\frac{1}{2} V\left(\langle\phi\rangle^{2}\right)+\left.2 \xi\langle\phi\rangle \frac{\partial V}{\partial \phi}\right|_{\phi=\langle\phi\rangle}+\Lambda_{(5)} \\
+\left.K^{2}\right|_{\phi=\langle\phi\rangle}\left[\left(\frac{1}{\kappa_{(5)}^{2}}+\xi\langle\phi\rangle^{2}\right) \frac{d^{2}-d+4}{2 d^{2}}+(2 \xi\langle\phi\rangle)^{2}\left(\frac{-d^{2}+3 d+4}{2 d^{2}}-8 \xi\right)\right] .
\end{gathered}
$$

Moreover, imposing that the effective cosmological vanishes on the brane, then

$$
\begin{aligned}
& \Lambda_{(5)}=-\frac{1}{2} V\left(\langle\phi\rangle^{2}\right)-\left.2 \xi\langle\phi\rangle \frac{\partial V}{\partial \phi}\right|_{\phi=\langle\phi\rangle} \\
&-\left.K^{2}\right|_{\phi=\langle\phi\rangle}\left[\left(\frac{1}{\kappa_{(5)}^{2}}+\xi\langle\phi\rangle^{2}\right) \frac{d^{2}-d+4}{2 d^{2}}+(2 \xi\langle\phi\rangle)^{2}\left(\frac{-d^{2}+3 d+4}{2 d^{2}}-8 \xi\right)\right] .
\end{aligned}
$$


We thus observe that a non-vanishing vev in the bulk generates in the gravitational sector a contribution to the cosmological constant on the brane.

\section{B. The effective potential}

Whether a non-vanishing vev for the scalar field can be observed on the brane depends on the form of the effective potential $V_{e f f}\left(\phi^{2}\right)$. The parameters of the potential will influence the magnitude of its minimum and consequently the mass of the scalar field $\phi$ measured on the brane, defined as the value of the second derivative of the effective potential evaluated at the vev of the scalar field, $\langle\phi\rangle$. We first determine the effective potential measured on the brane and then proceed to study the conditions for a non-vanishing vev.

The evolution of $\phi$ on the brane, as described by Eq. (21),

$$
g^{A B} \nabla_{A} \nabla_{B} \phi-\frac{\partial V_{e f f}}{\partial \phi}+\left(\frac{1}{4}+2 \xi\right)\left(\nabla_{C} \phi\right)^{2} \frac{2 \xi \phi}{1 / \kappa_{(5)}^{2}+\xi \phi^{2}(1+4 \xi)}=0
$$

is determined by a damping term as well as by the effective potential induced on the brane. Here,

$$
\begin{aligned}
-\frac{\partial V_{e f f}}{\partial \phi} & =\frac{1}{1 / \kappa_{(5)}^{2}+\xi \phi^{2}(1+4 \xi)}\left\{-\frac{\partial V}{\partial \phi}\left(\frac{1}{\kappa_{(5)}^{2}}+\xi \phi^{2}\right)\right. \\
& +2 \xi \phi\left[\frac{1}{2} V+\Lambda_{(5)}\right] \\
& \left.+2 \xi \phi K^{2}\left[\frac{1}{\kappa_{(5)}^{2}}\left(-\frac{2}{d}+2+8 \xi\right)+\xi \phi^{2}\left(-\frac{2}{d}+2+12 \xi\right)\right]\right\}
\end{aligned}
$$

where $V\left(\phi^{2}\right)$ is the bulk potential, which is assumed to have a Higgs type form $V(\phi)=$ $\mu_{(5)}^{2}\left(\phi^{2} / 2\right)+\lambda_{(5)}\left(\phi^{4} / 4\right)$ with $\lambda_{(5)}>0$. We compute $V_{\text {eff }}$ by integrating Eq. (26) to find that

$$
\begin{aligned}
V_{e f f}\left(\phi^{2}\right)= & \phi^{2}\left[\frac{\mu_{(5)}^{2}}{2} \frac{1}{1+4 \xi}-\frac{\mu_{(5)}^{2}}{4} \frac{1}{1+4 \xi}+\lambda_{(5)} \frac{1}{\xi \kappa_{(5)}^{2}} \frac{1}{2(1+4 \xi)^{2}}\left(\frac{1}{4}+4 \xi\right)\right] \\
+ & \phi^{4} \frac{\lambda_{(5)}}{4}\left[\frac{1}{1+4 \xi}-\frac{1}{4(1+4 \xi)}\right] \\
+ & \ln \left[1+\xi \kappa_{(5)}^{2} \phi^{2}(1+4 \xi)\right] \times \\
& \times\left[\mu_{(5)}^{2} \frac{1}{\xi \kappa_{(5)}^{2}} \frac{1}{2(1+4 \xi)^{2}}\left(\frac{1}{2}+4 \xi\right)-\lambda_{(5)} \frac{1}{\left(\xi \kappa_{(5)}^{2}\right)^{2}} \frac{1}{2(1+4 \xi)^{3}}\left(\frac{1}{4}+4 \xi\right)\right. \\
& \left.-\frac{1}{1+4 \xi} \Lambda_{(5)}\right] \\
+ & \frac{\sigma^{2}}{4} \frac{d^{2}}{(d+1)(d-1)} \times
\end{aligned}
$$




$$
\begin{aligned}
& \times\left[\frac{[-2 / d+2(1+4 \xi)](8 d /(d-1))-4}{1+8 \xi d /(d-1)} \frac{1}{1 / \kappa_{(5)}^{2}+\xi \phi^{2}[1+8 \xi d /(d-1)]}\right. \\
& \left.-\frac{\kappa_{(5)}^{2}}{\xi} \frac{d-1}{d+1}\left(-\frac{2}{d}+1+8 \xi\right) \ln \left[\frac{1+\xi \kappa_{(5)}^{2} \phi^{2}(1+4 \xi)}{1+\xi \kappa_{(5)}^{2} \phi^{2}[1+8 \xi d /(d-1)]}\right]\right]
\end{aligned}
$$

for $\xi \neq-1 / 4$. Notice that, in the limit when $\xi \rightarrow 0$, one recovers the original bulk potential with a term on the brane tension, $V\left(\phi^{2}\right)+3 \sigma^{2} \kappa_{(5)}^{2} d^{2} /[(d+1)(d-1)]$. It is natural to expect that there exists a hierarchy of scales depending on whether the vev $\langle\phi\rangle$ is related to the Standard Model (SM) scale or the grand unified theory scale. Thus, for $\left|\xi \phi^{2}\right| \ll 1 / \kappa_{(5)}^{2}$, we can expand the denominator of the first term in $\sigma$ about $1 / \kappa_{(5)}^{2}$, keeping terms up to order six in $\phi,\left(\xi \phi^{2}\right)^{3}$. The effective potential can thus be written as

$$
\begin{aligned}
V_{e f f}\left(\phi^{2}\right)= & \phi^{2}\left\{\frac{\mu_{(5)}^{2}}{2} \frac{1}{1+4 \xi}-\frac{\mu_{(5)}^{2}}{4} \frac{1}{1+4 \xi}+\lambda_{(5)} \frac{1}{\xi \kappa_{(5)}^{2}} \frac{1}{2(1+4 \xi)}\left(\frac{1}{4}+4 \xi\right)\right. \\
& \left.-\frac{1}{4} \sigma^{2} \kappa_{(5)}^{2}\left(\xi \kappa_{(5)}^{2}\right) \frac{d^{2}}{(d+1)(d-1)}\left[\left(-\frac{2}{d}+2(1+4 \xi)\right) \frac{8 d}{d-1}-4\right]\right\} \\
+ & \phi^{4}\left\{\frac{\lambda_{(5)}}{4} \frac{1}{1+4 \xi}-\frac{\lambda(5)}{4} \frac{1}{4(1+4 \xi)}\right. \\
& \left.+\frac{1}{4} \sigma^{2} \kappa_{(5)}^{2} \frac{1}{2}\left(\xi \kappa_{(5)}^{2}\right)^{2} \frac{d^{2}}{(d+1)(d-1)}\left[\left(-\frac{2}{d}+2(1+4 \xi)\right) \frac{8 d}{d-1}-4\right]\left(1+\xi \frac{8 d}{d-1}\right)\right\} \\
- & O\left[\phi^{6}\right] \frac{1}{4} \sigma^{2} \kappa_{(5)}^{2} \frac{1}{6}\left(\xi \kappa_{(5)}^{2}\right)^{3} \\
+ & \ln \left[1+\xi \kappa_{(5)}^{2} \phi^{2}(1+4 \xi)\right] \times \\
& \times\left[\mu_{(5)}^{2} \frac{1}{\xi \kappa_{(5)}^{2}} \frac{1}{2(1+4 \xi)^{2}}\left(\frac{1}{2}+4 \xi\right)-\lambda(5) \frac{1}{\left(\xi \kappa_{(5)}^{2}\right)^{2}} \frac{1}{2(1+4 \xi)^{3}}\left(\frac{1}{4}+4 \xi\right)\right. \\
& \left.-\frac{1}{1+4 \xi} \Lambda_{(5)}\right] \\
+ & \frac{1}{4} \sigma^{2} \kappa_{(5)}^{2} \frac{d^{2}}{(d+1)(d-1)} \times \\
& \times\left[\frac{[-2 / d+2(1+4 \xi)](8 d /(d-1))-4}{1+8 \xi d /(d-1)}\right. \\
& \left.-\frac{1}{\xi} \frac{d-1}{d+1}\left(-\frac{2}{d}+1+8 \xi\right) \ln \left[\frac{1+\xi \kappa_{(5)}^{2} \phi^{2}(1+4 \xi)}{1+\xi \kappa_{(5)}^{2} \phi^{2}[1+8 \xi d /(d-1)]}\right]\right] .
\end{aligned}
$$

We thus observe that, up to sub-dominant logarithmic terms, the effective potential is of the form $V_{e f f}=\mu_{e f f}^{2}\left(\phi^{2} / 2\right)+\lambda_{e f f}\left(\phi^{4} / 4\right)+O\left[\phi^{6}\right]$, where

$$
\begin{aligned}
& \mu_{e f f}^{2} \sim \mu_{(5)}^{2}+\lambda_{(5)} \frac{1}{\xi \kappa_{(5)}^{2}}-\sigma^{2} \xi \kappa_{(5)}^{4}, \\
& \lambda_{e f f} \sim \lambda_{(5)}+\sigma^{2} \xi^{2} \kappa_{(5)}^{6} .
\end{aligned}
$$

If $\mu_{e f f}^{2}<0$ and $\lambda_{e f f}>0$, then one expects a non-vanishing vev for the scalar field. The first condition guarantees that a non-vanishing minimum exists, whereas the second 
condition guarantees that such minimum is finite. Conversely, if $\mu_{\text {eff }}^{2}>0$ and $\lambda_{\text {eff }}>0$, then symmetry is always unbroken. Thus, imposing that $\lambda_{\text {eff }}>0$, it follows that $\lambda_{(5)}>$ $-\sigma^{2} \xi^{2} \kappa_{(5)}^{6}$. Consequently, in order not to spoil the $\mu_{e f f}^{2}<0$ condition we must have that $\mu_{(5)}^{2}<-2 \sigma^{2} \xi \kappa_{(5)}^{4}$.

We notice that the bulk scalar field $\phi$, being a five-dimensional field, has dimensions $[\phi]=M^{3 / 2}$. Accordingly, $\mu_{(5)}$ has dimensions of mass and $\lambda_{(5)}$ dimensions of inverse of mass. In order to recover characteristically four-dimensional quantities, we define the fourdimensional scalar field $\Phi$ as the rescaling of $\phi$ by an appropriate mass scale $M_{(\phi)}$. In the mode expansion of a bulk field, this mass can be identified with the mode function dependent on the direction $N$ evaluated at the position of the brane in the bulk. Thus, for $\phi=M_{\phi}^{\frac{1}{2}} \Phi$, the induced equation of motion for $\Phi$ on the brane becomes

$$
g^{A B} \nabla_{A} \nabla_{B} \Phi-\frac{1}{M_{\phi}} \frac{\partial V_{e f f}}{\partial \Phi}+\left(\frac{1}{4}+2 \xi\right)\left(\nabla_{C} \Phi\right)^{2} \frac{2 \xi M_{\phi} \Phi}{1 / \kappa_{(5)}^{2}+\xi M_{\phi} \Phi^{2}(1+4 \xi)}=0
$$

Consequently, the parameters of the effective potential will scale as

$$
\frac{1}{M_{\phi}} V_{e f f}\left(\Phi^{2}\right)=\mu_{e f f}^{2} \Phi^{2}+\lambda_{e f f} M_{\phi} \Phi^{4}+M_{\phi}^{2} O\left[\Phi^{6}\right] .
$$

with equations (29) and (30) becoming

$$
\begin{aligned}
\mu_{e f f}^{2} & \sim \mu^{2}-2 \sigma^{2} \xi \kappa_{(5)}^{4}, \\
M_{\phi} \lambda_{e f f} & \sim \lambda+M_{\phi} \xi^{2} \sigma^{2} \kappa_{(5)}^{6}
\end{aligned}
$$

where $\mu=\mu_{(5)}$ and $\lambda=M_{\phi} \lambda_{(5)}$. Here, for $\xi>0$ we have two possible mechanisms for the generation of a non-vanishing vev: the canonical way, via the potential associated with the scalar field, and the braneworld way, via the interaction of the scalar field with the brane tension. For the latter to be viable in the context of the SM, then

$$
\left|\frac{\mu_{e f f}^{2}}{M_{\phi} \lambda_{e f f}}\right| \sim \frac{1}{\xi M_{\phi}} \frac{1}{\kappa_{(5)}^{2}}
$$

must be of order $T e V^{2}$, and $\langle\Phi\rangle=246 \mathrm{GeV}$. However, in order to recover the fourdimensional gravitational coupling constant in Eq. (22), we find from the $\phi$ contribution that $M_{P l(4)}^{-2}=\kappa_{(5)}^{2} M_{\phi}$ and from the $\sigma$ contribution that $M_{P l(4)}^{-2} \sim \kappa_{(5)}^{4} \sigma$. It follows that

$$
M_{P l}^{3} \equiv \frac{1}{\kappa_{(5)}^{2}} \sim \frac{\sigma}{M_{\phi}}
$$

with $\sigma \sim M_{P l(4)}^{2} M_{\phi}^{2}$. This implies that

$$
\left|\frac{\mu_{e f f}^{2}}{M_{\phi} \lambda_{e f f}}\right| \sim \frac{1}{\xi} M_{P l(4)}^{2} \gg T e V^{2},
$$


which renders the brane mediated mechanism of SSB unviable for phenomenological reasons. This means that the phenomenological hierarchy between the SM typical energy scale order $\mathrm{TeV}$ and the Planck scale of the gravitational effects of the physics on the brane cannot be powered by the brane mediated mechanism of SSB, since the characteristic scale of the induced dynamics of the scalar field is the Planck scale. It is easy to see that $\langle\Phi\rangle \sim M_{P l(4)}$. Thus, the scalar field becomes a short range field about the brane and therefore strongly localized therein.

Moreover, from the expression for the five-dimensional cosmological in the case of a vanishing effective cosmological constant, Eq. (24), we find that $\Lambda_{(5)} \sim-M_{\phi} V\left(\langle\Phi\rangle^{2}\right)-$ $\sigma^{2} \kappa_{(5)}^{2}$, and consequently that

$$
M_{P l}^{3} \equiv \frac{1}{\kappa_{(5)}^{2}} \sim \frac{\sigma^{2}}{-\Lambda_{(5)}-M_{\phi} V\left(\langle\Phi\rangle^{2}\right)} .
$$

It then follows that $-\Lambda_{(5)}-M_{\phi} V\left(\langle\Phi\rangle^{2}\right) \sim M_{P l(4)}^{2} M_{\phi}^{3}$.

The vev of $\phi$ is localized at the minimum of the effective potential, $\left(\partial V_{e f f} / \partial \phi\right)=0$. Only real and positive solutions for $\langle\phi\rangle$ are physically relevant. It is possible to show that, besides $\langle\Phi\rangle=0$, of the four real solutions only one is positive for all values of the equation parameters is hence physically acceptable.

\section{CHARGED SCALAR FIELD}

In this section, in order to study spontaneous symmetry breaking, we introduce a minimal coupling of a scalar field $\psi$ to a bulk $U(1)$ gauge potential $\mathbf{B}$ through the covariant derivative $\tilde{\nabla}_{\mu}=\nabla_{\mu}+i e B_{\mu}$. However, only the scalar field is coupled non-minimally to the graviton. For the invariance of the action under local gauge transformations, the scalar field $\psi$ must be complex. The coupling constant $e$ is a measure of the charge of the scalar field associated with the field generated by the gauge potential. In order to avoid that flux of the gauge field leaks to the bulk spacetime, the gauge symmetry must be broken and the field thus confined to the brane. However, the dynamics of the symmetry breaking cannot be fully understood before properly treating the field in the bulk and studying how it appears from the point of view of the brane.

The Lagrangian density consists of the terms introduced in the previous section, Eq. (1), 
and additionally of the kinetic term associated with the gauge potential, given as follows

$$
\mathcal{L}=\frac{1}{\kappa_{(5)}^{2}} R-2 \Lambda+\xi \psi^{2} R-g^{\mu \nu}\left(\tilde{\nabla}_{\mu} \psi\right)\left(\tilde{\nabla}_{\nu} \psi^{*}\right)-V\left(\psi^{2}\right)-\frac{1}{4} B_{\mu \nu} B^{\mu \nu}
$$

Here, $\psi^{2}=\psi \psi^{*}$ and $B_{\mu \nu}=\partial_{\mu} B_{\nu}-\partial_{\nu} B_{\mu}$ is the field strength tensor associated with $\mathbf{B}$.

\section{A. The induced dynamics}

First we work out how this bulk theory is observed on the brane. By varying the action with respect to the metric, we obtain the Einstein equation in the bulk

$$
\left(\frac{1}{\kappa_{(5)}^{2}}+\xi \psi^{2}\right) G_{\mu \nu}+\Lambda g_{\mu \nu}=\frac{1}{2} T_{\mu \nu}^{(\psi)}+\frac{1}{2} T_{\mu \nu}^{(B)}+\xi \Sigma_{\mu \nu}^{(\psi)},
$$

where

$$
\begin{aligned}
& T_{\mu \nu}^{(\psi)}=2\left(\tilde{\nabla}_{\mu} \psi\right)\left(\tilde{\nabla}_{\nu} \psi^{*}\right)+g_{\mu \nu}\left[-g^{\alpha \beta}\left(\tilde{\nabla}_{\alpha} \psi\right)\left(\tilde{\nabla}_{\beta} \psi^{*}\right)-V\left(\psi^{2}\right)\right], \\
& T_{\mu \nu}^{(B)}=B_{\mu \rho} B_{\nu}{ }^{\rho}-\frac{1}{4} g_{\mu \nu} B_{\rho \sigma} B^{\rho \sigma}
\end{aligned}
$$

are the stress-energy tensors associated with the fields $\psi$ and $\mathbf{B}$ respectively, and

$$
\begin{aligned}
\Sigma_{\mu \nu}^{(\psi)} & =\psi \nabla_{\mu} \nabla_{\nu} \psi^{*}+\psi^{*} \nabla_{\mu} \nabla_{\nu} \psi+\left(\nabla_{\mu} \psi\right)\left(\nabla_{\nu} \psi^{*}\right)+\left(\nabla_{\mu} \psi^{*}\right)\left(\nabla_{\nu} \psi\right) \\
& -g_{\mu \nu} g^{\alpha \beta}\left[\psi \nabla_{\alpha} \nabla_{\beta} \psi^{*}+\psi^{*} \nabla_{\alpha} \nabla_{\beta} \psi+2\left(\nabla_{\alpha} \psi\right)\left(\nabla_{\beta} \psi^{*}\right)\right]
\end{aligned}
$$

is the contribution from the $\psi$-graviton interaction term.

We now proceed to derive the induced Einstein equation. First we project the source terms in Eq. (40) along the parallel and orthogonal directions to the surface of the brane. The components are as follows for $T_{\mu \nu}^{(\psi)}$

$$
\begin{aligned}
T_{A B}^{(\psi)} & =2\left(\nabla_{A}+i e B_{A}\right) \psi\left(\nabla_{B}-i e B_{B}\right) \psi^{*} \\
& -g_{A B}\left[\left(\nabla_{C}+i e B_{C}\right) \psi\left(\nabla_{C}-i e B_{C}\right) \psi^{*}+\left(\nabla_{N}+i e B_{N}\right) \psi\left(\nabla_{N}-i e B_{N}\right) \psi^{*}+V\right], \\
T_{A N}^{(\psi)} & =2\left(\nabla_{A}+i e B_{A}\right) \psi\left(\nabla_{N}-i e B_{N}\right) \psi^{*}, \\
T_{N N}^{(\psi)} & =-\left(\nabla_{C}+i e B_{C}\right) \psi\left(\nabla_{C}-i e B_{C}\right) \psi^{*}+\left(\nabla_{N}+i e B_{N}\right) \psi\left(\nabla_{N}-i e B_{N}\right) \psi^{*}-V,
\end{aligned}
$$

for $T_{\mu \nu}^{(B)}$

$$
\begin{aligned}
T_{A B}^{(B)} & =\left(\nabla_{A} B_{C}-\nabla_{C} B_{A}\right)\left(\nabla_{B} B_{C}-\nabla_{C} B_{B}\right)+\left(\nabla_{A} B_{N}-\nabla_{N} B_{A}\right)\left(\nabla_{B} B_{N}-\nabla_{N} B_{B}\right) \\
& -\frac{1}{4} g_{A B}\left[\left(\nabla_{C} B_{D}-\nabla_{D} B_{C}\right)^{2}+2\left(\nabla_{N} B_{D}-\nabla_{D} B_{N}\right)^{2}\right], \\
T_{A N}^{(B)} & =\left(\nabla_{A} B_{C}-\nabla_{C} B_{A}\right)\left(\nabla_{N} B_{C}-\nabla_{C} B_{N}\right),
\end{aligned}
$$




$$
T_{N N}^{(B)}=-\frac{1}{4}\left[\left(\nabla_{C} B_{D}-\nabla_{D} B_{C}\right)^{2}-2\left(\nabla_{N} B_{D}-\nabla_{D} B_{N}\right)^{2}\right]
$$

and for $\Sigma_{\mu \nu}^{(\psi)}$

$$
\begin{aligned}
\Sigma_{A B}^{(\psi)}= & \psi\left(\nabla_{A} \nabla_{B}+K_{A B} \nabla_{N}\right) \psi^{*}+\psi^{*}\left(\nabla_{A} \nabla_{B}+K_{A B} \nabla_{N}\right) \psi+\left(\nabla_{A} \psi\right)\left(\nabla_{B} \psi^{*}\right)+\left(\nabla_{A} \psi^{*}\right)\left(\nabla_{B} \psi\right) \\
- & g_{A B}\left[\psi\left(\nabla_{C}^{2}+K \nabla_{N}+\nabla_{N}^{2}\right) \psi^{*}+\psi^{*}\left(\nabla_{C}^{2}+K \nabla_{N}+\nabla_{N}^{2}\right) \psi\right. \\
& \left.+2\left(\nabla_{C} \psi\right)\left(\nabla_{C} \psi^{*}\right)+2\left(\nabla_{N} \psi\right)\left(\nabla_{N} \psi^{*}\right)\right] \\
\Sigma_{A N}^{(\psi)}= & \psi\left(\nabla_{A} \nabla_{N}-K_{A B} \nabla_{B}\right) \psi^{*}+\psi^{*}\left(\nabla_{A} \nabla_{N}-K_{A B} \nabla_{B}\right) \psi+\left(\nabla_{A} \psi\right)\left(\nabla_{N} \psi^{*}\right)+\left(\nabla_{A} \psi^{*}\right)\left(\nabla_{N} \psi\right), \\
\Sigma_{N N}^{(\psi)}= & -\left[\psi\left(\nabla_{C}^{2}+K \nabla_{N}\right) \psi^{*}+\psi^{*}\left(\nabla_{C}^{2}+K \nabla_{N}\right) \psi+2\left(\nabla_{C} \psi\right)\left(\nabla_{C} \psi^{*}\right)\right] .
\end{aligned}
$$

We equate the $(A B)$ components of the decomposition of the Einstein tensor and of the source terms from both the scalar field and the gauge potential to obtain the $(A B)$ component of the Einstein equation

$$
\begin{aligned}
& \quad\left(\frac{1}{\kappa_{(5)}^{2}}+\xi \psi^{2}\right)\left[G_{A B}^{(i n d)}+2 K_{A C} K_{C B}-K_{A B} K-K_{A B, N}-\frac{1}{2} g_{A B}\left(-K_{C D} K_{D C}-K^{2}-2 K_{, N}\right)\right] \\
& +g_{A B} \Lambda=\frac{1}{2} T_{A B}^{(\psi)}+\frac{1}{2} T_{A B}^{(B)}+\xi \Sigma_{A B}^{(\psi)} .
\end{aligned}
$$

Next we proceed to integrate the $(A B)$ component of the Einstein equation in the coordinate normal to the brane to obtain the matching conditions for the extrinsic curvature across the brane. We find that

$$
\left[\left(\frac{1}{\kappa_{(5)}^{2}}+\xi \psi^{2}\right)\left(-K_{A B}+g_{A B} K\right)\right]_{-\delta}^{+\delta}=\int_{-\delta}^{+\delta} d N\left[-g_{A B} \Lambda_{(4)}\right]-g_{A B} \xi\left[\psi \nabla_{N} \psi^{*}+\psi^{*} \nabla_{N} \psi\right]_{-\delta}^{+\delta}
$$

which yields

$$
\left(\frac{1}{\kappa_{(5)}^{2}}+\xi \psi^{2}\right)\left(-K_{A B}+g_{A B} K\right)=-g_{A B}\left[\sigma+\xi\left(\psi \nabla_{N} \psi^{*}+\psi^{*} \nabla_{N} \psi\right)\right]
$$

These provide ten boundary conditions for the extrinsic curvature on the brane. As noticed in the previous section, equating the $(A N)$ components of the Einstein equation we find four constraint conditions on the extrinsic curvature, $K_{A B ; B}-K_{; A}=0$, which follow from the conservation of the stress-energy tensor induced on the brane. The $(N N)$ equation

$$
\left(\frac{1}{\kappa_{(5)}^{2}}+\xi \psi^{2}\right)\left[-R^{(i n d)}-K_{C D} K_{C D}+K^{2}\right]+\Lambda_{(5)}=\frac{1}{2} T_{N N}^{(\psi)}+\frac{1}{2} T_{N N}^{(B)}+\xi \Sigma_{N N}^{(\psi)}
$$

provides the remaining condition relating the intrinsic curvature of the induced metric with the stress-energy sources. 
To derive the equation of motion for the scalar field we vary the action with respect to $\psi$ and $\psi^{*}$, finding respectively for $\psi^{*}$ the equation

$$
g^{\mu \nu}\left[\nabla_{\mu} \nabla_{\nu}-2 i e B_{\mu} \nabla_{\nu}+\left(i e B_{\mu}\right)\left(i e B_{\nu}\right)-i e\left(\nabla_{\mu} B_{\nu}\right)\right] \psi^{*}-\frac{\partial V}{\partial \psi}+\xi \psi^{*} R=0
$$

and for $\psi$ the corresponding complex conjugate equation

$$
g^{\mu \nu}\left[\nabla_{\mu} \nabla_{\nu}+2 i e B_{\mu} \nabla_{\nu}+\left(i e B_{\mu}\right)\left(i e B_{\nu}\right)+i e\left(\nabla_{\mu} B_{\nu}\right)\right] \psi-\frac{\partial V}{\partial \psi^{*}}+\xi \psi R=0 .
$$

We rewrite these equations by expanding the tensor quantities as follows

$$
\begin{aligned}
& {\left[g^{A B}\left(\nabla_{A} \nabla_{B}+K_{A B} \nabla_{N}\right)+\nabla_{N} \nabla_{N}-2 i e\left(g^{A B} B_{A} \nabla_{B}+B_{N} \nabla_{N}\right)\right.} \\
+ & \left.g^{A B}\left(i e B_{A}\right)\left(i e B_{B}\right)+\left(i e B_{N}\right)\left(i e B_{N}\right)-i e g^{A B}\left(\nabla_{A} B_{B}+K_{A B} B_{N}\right)-i e \nabla_{N} B_{N}\right] \psi^{*} \\
- & \frac{\partial V}{\partial \psi}+\xi \psi^{*}\left(R^{(i n d)}-K_{A B} K_{B A}-K^{2}-2 K_{, N}\right)=0,
\end{aligned}
$$

and equivalently for the corresponding complex conjugate. We now integrate in the $N$ direction to find the matching condition for $\psi^{*}$

$$
\int_{-\delta}^{+\delta} d N\left[\left(K \nabla_{N}+\nabla_{N} \nabla_{N}-i e B_{N} \nabla_{N}-i e \nabla_{N} B_{N}\right) \psi^{*}-2 \xi \psi^{*} K_{, N}\right]=0,
$$

which yields

$$
\left[-2 \xi K \psi^{*}+\left(\nabla_{N}-i e B_{N}\right) \psi^{*}\right]_{-\delta}^{+\delta}=0
$$

Substituting back into Eq. (51) and eliminating the $\nabla_{N} \psi^{*}$ term, we obtain

$$
\begin{aligned}
& g^{A B}\left[\nabla_{A} \nabla_{B}-2 i e B_{A} \nabla_{B}+\left(i e B_{A}\right)\left(i e B_{B}\right)-i e \nabla_{A} B_{B}\right] \psi^{*} \\
- & \frac{\partial V}{\partial \psi}+\xi \psi^{*}\left(R^{(i n d)}-K_{A B} K_{B A}+(1+4 \xi) K^{2}\right)=0
\end{aligned}
$$

where the extrinsic curvature results from the matching condition in Eq. (49)

$$
K_{A B}=-g_{A B} \sigma \frac{1 /(d-1)}{1 / \kappa_{(5)}^{2}+\xi \psi^{2}(1+4 \xi d /(d-1))}
$$

and the intrinsic curvature results from the $(N N)$ component of the Einstein equation in Eq. (50)

$$
\begin{aligned}
& \left(\frac{1}{\kappa_{(5)}^{2}}+\xi \psi^{2}(1+2 \xi)\right) R^{(i n d)}=\left(\frac{1}{2}+2 \xi\right)\left(\nabla_{C}+i e B_{C}\right) \psi\left(\nabla_{C}-i e B_{C}\right) \psi^{*} \\
+ & \frac{1}{2} V+\xi\left(\psi \frac{\partial V}{\partial \psi}+\psi^{*} \frac{\partial V}{\partial \psi^{*}}\right)+\frac{1}{8}\left(\nabla_{C} B_{D}-\nabla_{D} B_{C}\right)
\end{aligned}
$$




$$
+\Lambda_{(5)}-K_{C D} K_{C D}\left(\frac{1}{\kappa_{(5)}^{2}}+\xi \psi^{2}(1-2 \xi)\right)+K^{2}\left(\frac{1}{\kappa_{(5)}^{2}}+\xi \psi^{2}\left(1-8 \xi^{2}\right)\right) .
$$

The matching condition and the induced equation of motion for $\psi$ are the complex conjugate of the corresponding equations for $\psi^{*}$.

For the equation of motion for the vector field $\mathbf{B}$, obtained by varying the action with respect to $B_{\mu}$, we find that

$$
\nabla^{\nu}\left(\nabla_{\nu} B_{\mu}-\nabla_{\mu} B_{\nu}\right)+i e g_{\mu \nu}\left(\psi^{*} \tilde{\nabla}^{\nu} \psi-\psi \tilde{\nabla}^{\nu} \psi^{*}\right)=0
$$

We project the equation parallel and orthogonal to the surface of the brane to find for the parallel component that

$$
\begin{aligned}
& \nabla_{C}\left(\nabla_{C} B_{A}-\nabla_{A} B_{C}\right)+\nabla_{N}\left(\nabla_{N} B_{A}-\nabla_{A} B_{N}\right) \\
+ & 2 K_{A C}\left(\nabla_{C} B_{N}-\nabla_{N} B_{C}\right)+K\left(\nabla_{N} B_{A}-\nabla_{A} B_{N}\right) \\
+ & i e\left(\psi^{*} \nabla_{A} \psi-\psi \nabla_{A} \psi^{*}\right)+(i e)^{2} B_{A} \psi^{2}=0
\end{aligned}
$$

and for the orthogonal component that

$$
\nabla_{C}\left(\nabla_{C} B_{N}-\nabla_{N} B_{C}\right)+i e\left(\psi^{*} \nabla_{N} \psi-\psi \nabla_{N} \psi^{*}\right)+(i e)^{2} B_{N} \psi^{2}=0
$$

Integrating Eqs. (60) and (61) in the $N$ direction, we find the matching conditions respectively for $B_{A}$

$$
\left[\nabla_{N} B_{A}-\nabla_{A} B_{N}\right]_{-\delta}^{+\delta}=0
$$

and for $B_{N}$

$$
\left[\nabla_{C} B_{C}-K B_{N}\right]_{-\delta}^{+\delta}=0
$$

We then substitute Eq. (62) in Eq. (60), obtaining for the induced $B_{A}$ equation

$$
\nabla_{C}\left(\nabla_{C} B_{A}-\nabla_{A} B_{C}\right)+i e\left(\psi^{*} \nabla_{A} \psi-\psi \nabla_{A} \psi^{*}\right)+(i e)^{2} B_{A} \psi^{2}=0
$$

We notice that the component $B_{A}$ will acquire on the brane a mass proportional to the scalar field vev. When $\psi$ acquires a non-vanishing vev, gauge invariance is spontaneously broken on the brane for $B_{A}$, which thus becomes a short range field about the brane.

To derive the induced $B_{N}$ equation we similarly substitute Eq. (63) in Eq. (61) and moreover use the matching conditions for the scalar field, Eq. (55) and its complex conjugate, obtaining

$$
\nabla_{C} \nabla_{C} B_{N}-K B_{N, N}-K_{C D} K_{D C} B_{N}=0
$$


We notice that on the brane $B_{N}$ does not interact with the scalar field but only with the brane tension, which is manifest in the vanishing coupling constant to $\psi$ induced on the brane. Consequently, in the case where $\psi$ acquires a non-vanishing vev, $B_{N}$ does not undergo spontaneous symmetry breaking, thus remaining massless with respect to $\psi$. (The breaking of gauge symmetry for $B_{N}$ would require a non-minimal coupling of $\mathbf{B}$ to gravity, as discussed in Ref. [9].) However, the $B_{N}$ component acquires a mass with respect to the brane tension $\sigma$, which implies that $B_{N}$ is strictly localized on the brane.

Finally, we substitute the matching conditions for the extrinsic curvature, Eq. (49), in the $(A B)$ component of the Einstein equation and moreover use the matching conditions for the scalar field, Eq. (55), as well as for the vector field, Eqs. (62) and (63), to find for the Einstein equation induced on the brane that

$$
\begin{aligned}
& \left(\frac{1}{\kappa_{(5)}^{2}}+\xi \psi^{2}\right) G_{A B}^{(i n d)}= \\
= & \xi\left[\psi \nabla_{A} \nabla_{B} \psi^{*}+\psi^{*} \nabla_{A} \nabla_{B} \psi+\left(\nabla_{A} \psi\right)\left(\nabla_{B} \psi^{*}\right)+\left(\nabla_{A} \psi^{*}\right)\left(\nabla_{B} \psi\right)\right. \\
& \left.-g_{A B} g^{C D}\left(\psi \nabla_{C} \nabla_{D} \psi^{*}+\psi^{*} \nabla_{C} \nabla_{D} \psi+2\left(\nabla_{C} \psi\right)\left(\nabla_{D} \psi^{*}\right)\right)\right] \\
+ & {\left[\left(\nabla_{A}+i e B_{A}\right) \psi\left(\nabla_{B}-i e B_{B}\right) \psi^{*}\right.} \\
& \left.-\frac{1}{2} g_{A B}\left(g^{C D}\left(\nabla_{C}+i e B_{C}\right) \psi\left(\nabla_{D}-i e B_{D}\right) \psi^{*}+\frac{1}{2 V}+\Lambda_{(5)}\right)\right] \\
+ & \frac{1}{2}\left[\left(\nabla_{A} B_{C}-\nabla_{C} B_{A}\right)\left(\nabla_{B} B_{C}-\nabla_{C} B_{B}\right)\right. \\
& \left.-g_{A B} \frac{1}{4}\left(\nabla_{C} B_{D}-\nabla_{D} B_{C}\right)\left(\nabla_{C} B_{D}-\nabla_{D} B_{C}\right)\right] \\
- & g_{A B} K^{2}\left(\frac{1}{\kappa_{(5)}^{2}}+\xi \psi^{2}\right)\left\{\frac{d^{2}-d+4}{2 d^{2}}+2 \xi^{2} \psi^{2}\right\}
\end{aligned}
$$

which, upon substitution of the induced equations of motion for the scalar field, further reduces to

$$
\begin{aligned}
& G_{A B}^{(i n d)}= \\
= & \left(\frac{1}{\kappa_{(5)}^{2}}+\xi \psi^{2}\right)^{-1}\left[\left(\nabla_{A}+i e B_{A}\right) \psi\left(\nabla_{B}-i e B_{B}\right) \psi^{*}+\frac{1}{2}\left(\nabla_{A} B_{C}-\nabla_{C} B_{A}\right)\left(\nabla_{B} B_{C}-\nabla_{C} B_{B}\right)\right. \\
& \left.+\xi\left[\psi \nabla_{A} \nabla_{B} \psi^{*}+\psi^{*} \nabla_{A} \nabla_{B} \psi+\left(\nabla_{A} \psi\right)\left(\nabla_{B} \psi^{*}\right)+\left(\nabla_{A} \psi^{*}\right)\left(\nabla_{B} \psi\right)\right]\right] \\
- & g_{A B}\left[\left(\frac{1}{2}+2 \xi\right)\left(\nabla_{C}+i e B_{C}\right) \psi\left(\nabla_{C}-i e B_{C}\right) \psi^{*}+\frac{1}{8}\left(\nabla_{D} B_{C}-\nabla_{C} B_{D}\right)^{2}\right. \\
& \left.+\frac{1}{2} V+\Lambda_{(5)}+\xi\left(\psi \frac{\partial V}{\partial \psi}+\psi^{*} \frac{\partial V}{\partial \psi^{*}}\right)\right] \frac{1}{1 / \kappa_{(5)}^{2}+\xi \psi^{2}(1+2 \xi)} \\
- & g_{A B} K^{2}\left[\left(\frac{1}{\kappa_{(5)}^{2}}+\xi \psi^{2}\right) \frac{d^{2}-d+4}{2 d^{2}}+2 \xi^{2} \psi^{2}\left(\frac{-d^{2}+3 d+4}{2 d^{2}}-4 \xi\right)\right]
\end{aligned}
$$




$$
\times \frac{1}{1 / \kappa_{(5)}^{2}+\xi \psi^{2}(1+2 \xi)} .
$$

We now proceed to realize the case where the scalar field $\psi$ acquires a non-vanishing vev $\langle\psi\rangle$, with $\psi^{*}$ acquiring the corresponding conjugate value $\langle\psi\rangle^{*}$. In order to avoid the breaking of Lorentz symmetry, we must have that $\nabla_{A}\langle\psi\rangle=\nabla_{A}\langle\psi\rangle^{*}=0$ and $\langle\mathbf{B}\rangle=0$. Moreover, $\langle\mathbf{B}\rangle$ must not be allowed to vary in space but must instead be forced to take on the same value everywhere in the bulk, which implies both $\nabla_{A}\langle\mathbf{B}\rangle=0$ and $\nabla_{N}\langle\mathbf{B}\rangle=0$.

We can read off of the induced Einstein equation the effective cosmological constant on the brane, defined as the term proportional to the induced metric

$$
\begin{aligned}
& \Lambda_{\text {eff }}\left(\frac{1}{\kappa_{(5)}^{2}}+\xi\langle\psi\rangle^{2}(1+2 \xi)\right)=\frac{1}{2} V\left(\langle\psi\rangle^{2}\right)+\Lambda_{(5)}+\xi\left(\left.\langle\psi\rangle \frac{\partial V}{\partial \psi}\right|_{\psi=\langle\psi\rangle}+\left.\langle\psi\rangle^{*} \frac{\partial V}{\partial \psi^{*}}\right|_{\psi=\langle\psi\rangle}\right) \\
+ & \left.K^{2}\right|_{\psi=\langle\psi\rangle}\left[\left(\frac{1}{\kappa_{(5)}^{2}}+\xi\langle\psi\rangle^{2}\right) \frac{d^{2}-d+4}{2 d^{2}}+2 \xi^{2}\langle\psi\rangle^{2}\left(\frac{-d^{2}+3 d+4}{2 d^{2}}-4 \xi\right)\right] .
\end{aligned}
$$

\section{B. The effective potential}

We now proceed to determine the effective potential for the field $\psi$ and study the conditions for SSB, following the same reasoning as that for the real scalar field $\phi$.

The evolution of $\psi^{*}$ on the brane is described by

$$
\begin{aligned}
& g^{A B} \nabla_{A} \nabla_{B} \psi^{*}-\frac{\partial V_{e f f}}{\partial \psi} \\
+ & {\left[-\left(i e B_{C}\right) \nabla_{C} \psi^{*}\left(2 \frac{1}{\kappa_{(5)}^{2}}+\xi \psi^{2}\left(\frac{3}{2}+2 \xi\right)\right)\right.} \\
& \left.+\xi \psi^{*}\left[\left(\frac{1}{2}+2 \xi\right)\left\{\left(\nabla_{C} \psi\right)\left(\nabla_{C} \psi^{*}\right)-\left(i e B_{C}\right) \psi^{*}\left(\nabla_{C} \psi\right)\right\}\right]\right] \frac{1}{1 / \kappa_{(5)}^{2}+\xi \psi^{2}(1+2 \xi)}=0
\end{aligned}
$$

where

$$
\begin{aligned}
& -\frac{\partial V_{e f f}}{\partial \psi}=-i e\left(\nabla_{C} B_{C}\right) \psi^{*} \\
& +\left\{-\frac{\partial V}{\partial \psi}\left(\frac{1}{\kappa_{(5)}^{2}}+\xi \psi^{2}(1+\xi)\right)+\xi^{2} \psi^{*} \psi^{*} \frac{\partial V}{\partial \psi^{*}}\right. \\
& +\left(i e B_{C}\right)^{2} \psi^{*}\left(\frac{1}{\kappa_{(5)}^{2}}+\frac{1}{2} \xi \psi^{2}\right) \\
& +\xi \psi^{*}\left[\frac{1}{8}\left(\nabla_{C} B_{D}-\nabla_{D} B_{C}\right)^{2}+\frac{1}{2} V+\Lambda_{(5)}\right] \\
& \left.+\xi \psi^{*} K^{2}\left[\frac{1}{\kappa_{(5)}^{2}}\left(-\frac{2}{d}+2+4 \xi\right)+\xi \psi^{2}\left(-\frac{2}{d}+2+6 \xi\right)\right]\right\} \frac{1}{1 / \kappa_{(5)}^{2}+\xi \psi^{2}(1+2 \xi)}
\end{aligned}
$$


Assuming as before that $V\left(\psi \psi^{*}\right)=\mu_{(5)}^{2} \psi \psi^{*}+\lambda_{(5)}\left(\psi \psi^{*}\right)^{2}$, we integrate in $\psi$ to find for the effective potential

$$
\begin{aligned}
V_{e f f}\left(\psi^{2}\right)= & \psi^{2}\left[\frac{\mu_{(5)}^{2}}{2} \frac{1}{1+2 \xi}-\frac{\mu_{(5)}^{2}}{4} \frac{1}{1+2 \xi}+\lambda_{(5)} \frac{1}{\xi \kappa_{(5)}^{2}} \frac{1}{2(1+2 \xi)^{2}}\left(\frac{1}{4}+2 \xi\right)\right. \\
& \left.-\left(i e B_{C}\right)^{2} \frac{1}{2(1+2 \xi)}+i e\left(\nabla_{C} B_{C}\right)\right] \\
+ & \psi^{4} \frac{\lambda_{(5)}}{4}\left[\frac{1}{1+2 \xi}-\frac{1}{4(1+2 \xi)}\right] \\
+ & \ln \left[1+\psi^{2} \kappa_{(5)}^{2} \xi(1+2 \xi)\right] \times \\
& \times\left[\mu_{(5)}^{2} \frac{1}{\xi \kappa_{(5)}^{2}} \frac{1}{2(1+2 \xi)^{2}}\left(\frac{1}{2}+2 \xi\right)-\lambda_{(5)} \frac{1}{\left(\xi \kappa_{(5)}^{2}\right)^{2}} \frac{1}{2(1+2 \xi)^{3}}\left(\frac{1}{4}+2 \xi\right)\right. \\
& \left.-\frac{1}{\xi \kappa_{(5)}^{2}} \frac{1}{2(1+2 \xi)^{2}}\left(i e B_{C}\right)^{2}-\frac{1}{1+2 \xi}\left[\frac{1}{8}\left(\nabla_{C} B_{D}-\nabla_{D} B_{C}\right)^{2}+\Lambda_{(5)}\right]\right] \\
+ & \frac{\sigma^{2}}{2} \frac{d^{2}}{(d+1)(d-1)} \times \\
& \times\left[\frac{[-2 / d+2(1+2 \xi)](4 d /(d-1))-2}{1+4 \xi d /(d-1)} \frac{1}{1 / \kappa_{(5)}^{2}+\xi \psi^{2}[1+4 \xi d /(d-1)]}\right. \\
& -\frac{\left.\kappa_{(5)}^{2} \frac{d-1}{\xi}\left(-\frac{2}{d}+1+4 \xi\right) \ln \left[\frac{1+\xi \kappa_{(5)}^{2} \psi^{2}(1+2 \xi)}{1+\xi \kappa_{(5)}^{2} \psi^{2}[1+4 \xi d /(d-1)]}\right]\right]}{}
\end{aligned}
$$

We expand the denominator of the first term in $\sigma$, finding that we can express the effective potential as

$$
\begin{aligned}
V_{e f f}\left(\psi^{2}\right)= & \psi^{2}\left\{\frac{\mu_{(5)}^{2}}{2} \frac{1}{1+2 \xi}-\frac{\mu_{(5)}^{2}}{4} \frac{1}{1+2 \xi}+\lambda_{(5)} \frac{1}{\xi \kappa_{(5)}^{2}} \frac{1}{2(1+2 \xi)^{2}}\left(\frac{1}{4}+2 \xi\right)\right. \\
& -\left(i e B_{C}\right)^{2} \frac{1}{2(1+2 \xi)}+i e\left(\nabla_{C} B_{C}\right) \\
& \left.-\frac{1}{2} \sigma^{2} \kappa_{(5)}^{2}\left(\xi \kappa_{(5)}^{2}\right) \frac{d^{2}}{(d+1)(d-1)}\left[\left(-\frac{2}{d}+2(1+2 \xi)\right) \frac{4 d}{d-1}-2\right]\right\} \\
+ & \psi^{4}\left\{\frac{\lambda(5)}{4} \frac{1}{1+2 \xi}-\frac{\lambda(5)}{4} \frac{1}{4(1+2 \xi)}\right. \\
& \left.+\frac{1}{2} \sigma^{2} \kappa_{(5)}^{2} \frac{1}{2}\left(\xi \kappa_{(5)}^{2}\right)^{2} \frac{d^{2}}{(d+1)(d-1)}\left[\left(-\frac{2}{d}+2(1+2 \xi)\right) \frac{4 d}{d-1}-2\right]\left(1+\xi \frac{4 d}{d-1}\right)\right\} \\
+ & O\left[\psi^{6}\right] \sigma^{2} \kappa_{(5)}^{2} \frac{1}{6}\left(\xi \kappa_{(5)}^{2}\right)^{3} \\
+ & \ln \left[1+\psi^{2} \kappa_{(5)}^{2} \xi(1+2 \xi)\right] \times \\
& \times\left[\mu_{(5)}^{2} \frac{1}{\xi \kappa_{(5)}^{2}} \frac{1}{2(1+2 \xi)^{2}}\left(\frac{1}{2}+2 \xi\right)-\lambda_{(5)} \frac{1}{\left(\xi \kappa_{(5)}^{2}\right)^{2}} \frac{1}{2(1+2 \xi)^{3}}\left(\frac{1}{4}+2 \xi\right)\right. \\
& \left.-\frac{1}{\xi \kappa_{(5)}^{2}} \frac{1}{2(1+2 \xi)^{2}}\left(i e B_{C}\right)^{2}-\frac{1}{1+2 \xi}\left[\frac{1}{8}\left(\nabla_{C} B_{D}-\nabla_{D} B_{C}\right)^{2}+\Lambda_{(5)}\right]\right] \\
+ & \frac{1}{2} \sigma^{2} \kappa_{(5)}^{2} \frac{d^{2}}{(d+1)(d-1)} \times \\
& \times\left[\frac{[-2 / d+2(1+2 \xi)](4 d /(d-1))-2}{1+4 \xi d /(d-1)}\right. \\
& {\left[\frac{1}{2(d)}\right.}
\end{aligned}
$$




$$
\left.-\frac{1}{\xi} \frac{d-1}{d+1}\left(-\frac{2}{d}+1+4 \xi\right) \ln \left[\frac{1+\xi \kappa_{(5)}^{2} \psi^{2}(1+2 \xi)}{1+\xi \kappa_{(5)}^{2} \psi^{2}[1+4 \xi d /(d-1)]}\right]\right] .
$$

At the vev's of the bulk fields, the effective potential is, up to logarithmic terms, of the form $V_{e f f}=\mu_{e f f}^{2}\left(\psi \psi^{*} / 2\right)+\lambda_{e f f}\left(\psi \psi^{*}\right)^{2}+O\left[\left(\psi \psi^{*}\right)^{3}\right]$, where

$$
\begin{aligned}
\mu_{e f f}^{2} & \sim \mu_{(5)}^{2}-2 \sigma^{2} \xi \kappa_{(5)}^{4} \\
\lambda_{e f f} & \sim \lambda_{(5)}+\sigma^{2} \xi^{2} \kappa_{(5)}^{6} .
\end{aligned}
$$

Upon the scalar field taking a non-vanishing vev $\langle\psi\rangle$ (with its conjugate taking the corresponding conjugate vev $\left.\left\langle\psi^{*}\right\rangle\right)$ and the gauge field taking $\langle\mathbf{B}\rangle=0$, with $\nabla_{A}\langle\mathbf{B}\rangle=0$, we find from Eq. (67) the same relations for the four-dimensional Planck scale and the matter fields mass scales as those found in Section II. Consequently, for $\mu_{e f f}<0$ and $M_{\psi} \lambda_{e f f}>0$, where $M_{\psi}$ is such that $\psi=M_{\psi}^{\frac{1}{2}} \Psi$, we find that $\left|\mu_{e f f} /\left(M_{\psi} \lambda_{e f f}\right)\right|$ yields the same constraint relation between $\xi$ and $M_{P l(4)}$ as that found for the case of $\Phi$ in Eq. (37). From similar considerations, it follows that the scalar field vev's are of order the Planck scale, which implies that both the mass of the scalar field and the mass of $B_{A}$ generated by the SSB mechanism are also of order the Planck scale. The mass of $B_{N}$ is of order $\sigma^{2} \sim\left(M_{P l(4)}^{2} M_{\phi}^{2}\right)^{2}$. Whereas $\Phi$ and $B_{A}$ become strongly localized about the brane, $B_{N}$ becomes localized on the brane by the delta profile of the brane tension. Notice that, as before, the appearance of a new mass scale associated with the scalar field is quite natural given that in the bulk spacetime the self-interaction coupling constant, $\lambda_{(5)}$, is not dimensionless.

Moreover, the surviving contributions to the condition for a vanishing effective cosmological constant are

$$
\begin{gathered}
\Lambda_{(5)}=-\frac{1}{2} V\left(\langle\psi\rangle\left\langle\psi^{*}\right\rangle\right)-\xi\left(\langle\psi\rangle \frac{\partial V}{\partial \psi}+\left\langle\psi^{*}\right\rangle \frac{\partial V}{\partial \psi^{*}}\right)_{\psi=\langle\psi\rangle, \psi^{*}=\left\langle\psi^{*}\right\rangle} \\
-\left.K^{2}\right|_{\psi^{2}=\langle\psi\rangle^{2}}\left[\left(\frac{1}{\kappa_{(5)}^{2}}+\xi\langle\psi\rangle\left\langle\psi^{*}\right\rangle\right) \frac{d^{2}-d+4}{2 d^{2}}+2 \xi^{2}\langle\psi\rangle\left\langle\psi^{*}\right\rangle\left(\frac{-d^{2}+3 d+4}{2 d^{2}}-4 \xi\right)\right] .
\end{gathered}
$$

Hence, for a vanishing cosmological constant on the brane the five-dimensional Planck scale is found to scale like the condition in Eq. (38).

\section{DISCUSSION AND CONCLUSIONS}

In this work we have examined the mechanism of spontaneous symmetry breaking due to a scalar field in the bulk spacetime coupled non-minimally to gravity. We have shown 
that a bulk scalar field can be a source of symmetry breaking on the brane but only at very high energies. We derived the conditions which allow for the existence of a non-vanishing bulk scalar field vacuum configuration and demonstrated that the scales of the induced masses are of order the four-dimensional Planck scale, thus failing to accommodate on the brane the typical scales of the Standard Model. We notice, however, that this implies that the bulk scalar fields become very short range about the position of the brane and thus strongly localized.

In the presence of a bulk gauge field, we found that on the brane the component $B_{A}$ interacts with the charged scalar field, whereas the component $B_{N}$ interacts solely with the brane tension. This implies that, upon the acquisition by the scalar field of a nonvanishing vev, the component $B_{A}$ acquires a mass of order the Planck mass on the brane and thus becomes localized. The component $B_{N}$ remains massless with respect to the interaction with $\psi$, acquiring however a "topological" mass derived from the coupling with the delta-localized brane tension.

Furthermore, we observe that in the absence of the non-minimal coupling of the bulk scalar fields to gravity, i.e. for $\xi=0$, the effective potential on the brane of a bulk scalar field reduces in both cases to $V_{\text {eff }}=\mu_{(5)}^{2}\left(\phi^{2} / 2\right)+\lambda_{(5)}\left(\phi^{4} / 4\right) V+3 \sigma^{2} \kappa_{(5)}^{2} d^{2} /[(d+1)(d-1)]$. The realization of a braneworld universe as a co-dimension one surface of localized matter contributes a constant term proportional to the brane tension $\sigma$ to the effective potential. The brane tension does not, however, contribute to the mechanism of spontaneous symmetry breaking observed on the brane unless the bulk scalar fields are non-minimally coupled to gravity. This is observed in the dependence on the coupling parameter $\xi$ of the parameters $\mu_{e f f}^{2}$ and $\lambda_{e f f}$. Moreover, the mixing of the discontinuity in the extrinsic curvature with the discontinuity in the normal derivative of the scalar field, as encompassed by the corresponding matching conditions, is also $\xi$-dependent. Such mixing is switched off when $\xi=0$, as already noticed in Ref. [11] and also found in Ref. [9]. These observations seem to suggest that the matter localized on the brane will only interact with bulk matter fields through gravity when a non-minimal coupling exists.

On a more technical side, our approach goes a step further in setting up the framework for studying the brane induced physics which arises from the presence of matter fields in the bulk. The case of a vector field coupled non-minimally to gravity was previously considered and its implications for Lorentz symmetry on the brane studied in Ref. [9]. A scalar field coupled non-minimally to gravity is treated in the present paper. Our approach allows to 
relate the cosmological constant problem and the scale of gravity with the mechanism of origin of mass, which we have found to relate with the mechanism of localization of bulk fields on the brane.

\section{Acknowledgments}

C. C. thanks Fundação para a Ciência e a Tecnologia - FCT (Portuguese Agency) for financial support under the fellowship /BPD/18236/2004. O. B. acknowledges the partial support of the FCT project POCI/FP/63916/2005. The authors would like to thank Martin Bucher, Mariam Bouhmadi Lopez, Fernando Quevedo and Kyriakos Tamvakis for useful discussions.

[1] O. Bertolami, Stability Conditions For A Scalar Field Coupled Nonminimally With Gravity, Phys. Lett. B 186 (1987) 161.

[2] Y. Fujii, Scalar-tensor theory of gravitation and spontaneous breakdown of scale invariance, Phys. Rev. D 9 (1974) 874; A. Zee, The Horizon Problem And The Broken Symmetric Theory Of Gravity, Phys. Rev. Lett. 42 (1979) 417; Spontaneously Generated Gravity, Phys. Rev. D 23 (1981) 858; S.L. Adler, Einstein Gravity as a Symmetry Breaking Effect in Quantum Field Theory, Rev. Mod. Phys. 54 (1982) 729.

[3] J. Scherk and J.H. Schwarz, Dual models for non-hadrons, Nucl. Phys. B 81 (1974) 118; A. Sen, Equations of Motion for the Heterotic String Theory from the Conformal Invariance of the Sigma Model, Phys. Rev. Lett. 55 (1985) 1846; Heterotic string in an arbitrary background field, Phys. Rev. D 32 (1985) 2102; C.G. Callan, D. Friedan, E. Martinec and M.J. Perry, Strings in background fields, Nucl. Phys. B 262 (1985) 593; A.A. Tseytlin, Vector field effective action in the open superstring theory, Nucl. Phys. B 276 (1986) 391; D.J. Gross and E. Witten, Superstring modifications of Einstein's equations, Nucl. Phys. B 277 (1986) 1; R.R. Metsaev and A.A. Tseytlin, Order $\alpha^{\prime}$ (two-loop) equivalence of the string equations of motion and the $\sigma$-model Weyl invariance conditions : Dependence on the dilaton and the antisymmetric tensor, Nucl. Phys. B 293 (1987) 385; Curvature cubed terms in string theory effective actions, Phys. Lett. B 185 (1987) 52; M.C. Bento, O. Bertolami, A.B. Henriques and J.C. Romão, Order $\alpha^{\prime 2}$ terms in the gravitational sector of string effective actions with the inclusion of the dilaton field, Phys. Lett. B 218 (1989) 162; Order $\alpha^{\prime 2}$ equivalence of 
the string equations of motion and the $\sigma$-model Weyl invariance conditions: Dependence on the dilaton field, Phys. Lett. B 220 (1989) 113; M.C. Bento, O. Bertolami and J.C. Romão, Equivalente of the string equations of motion and the $\sigma$-model Weyl invariance conditions at order $\alpha^{\prime 2}$ : Dependence on the dilaton field, Int. J. Mod. Phys. A 6 (1991) 5099.

[4] G.R. Dvali, I.I. Kogan and M.A. Shifman, Topological effects in our brane world from extra dimensions, Phys. Rev. D62 (2000) 106001.

[5] A. Kehagias and K. Tamvakis, Bulk Higgs with 4-D gauge interactions, Phys. Lett. B 628 (2005) 262.

[6] W.D. Goldberger and M.B. Wise, Modulus stabilization with bulk fields, Phys. Rev. Lett. 83 (1999) 4922.

[7] S. Chang, J. Hisano, H. Nakano, N. Okada and M. Yamaguchi, Bulk standard model in the Randall-Sundrum background, Phys. Rev. D 62 (2000) 084025.

[8] M. Bucher and C. Carvalho, Linearized Israel matching conditions for cosmological perturbations in a moving brane background, Phys. Rev. D 71 (2005) 083511.

[9] O. Bertolami and C. Carvalho, Lorentz Symmetry Derived from Lorentz Violation in the Bulk, Phys. Rev. D 74 (2006) 084020 (see also O. Bertolami and C. Carvalho, gr-qc/0612129 for further discussions.)

[10] P.V. Moniz, P. Crawford and A. Barroso, Spontaneous symmetry breaking in curved spacetime, Class. Quantum Grav. 7 (1990) L143.

[11] C. Barceló, M. Visser, Moduli fields and brane tension: generalizing the junction conditions, Phys. Rev. D 63 (2001) 02400. 